\title{
Some Exact Results for Mid-Band and Zero Band-Gap States of Associated Lamé Potentials
}

\author{
Avinash Khare ${ }^{a, \text {, and Uday Sukhatme }}$, $^{b}$
}

a) Institute of Physics, Sachivalaya Marg, Bhubaneswar 751005, Orissa, India

b) Department of Physics, University of Illinois at Chicago, Chicago, IL 60607-7059, U.S.A.

\begin{abstract}
Applying certain known theorems about one-dimensional periodic potentials, we show that the energy spectrum of the associated Lamé potentials

$$
a(a+1) m \operatorname{sn}^{2}(x, m)+b(b+1) m \operatorname{cn}^{2}(x, m) / \operatorname{dn}^{2}(x, m)
$$

consists of a finite number of bound bands followed by a continuum band when both $a$ and $b$ take integer values. Further, if $a$ and $b$ are unequal integers, we show that there must exist some zero band-gap states, i.e. doubly degenerate states with the same number of nodes. More generally, in case $a$ and $b$ are not integers, but either $a+b$ or $a-b$ is an integer $(a \neq b)$, we again show that several of the band-gaps vanish due to degeneracy of states with the same number of nodes. Finally, when either $a$ or $b$ is an integer and the other takes a half-integral value, we obtain exact analytic solutions for several mid-band states.
\end{abstract}

\footnotetext{
${ }^{1}$ khare@iopb.res.in

${ }^{2}$ sukhatme@uic.edu
} 


\section{Introduction}

The energy spectrum of electrons on a lattice is of central importance in condensed matter physics. In particular, the knowledge of the existence and locations of band edges and band gaps determines many physical properties. Unfortunately, even in one dimension, there are very few analytically solvable periodic potential problems in quantum mechanics. The Lamé potential

$$
V(x)=a(a+1) m \operatorname{sn}^{2}(x, m), a=1,2,3, \ldots,
$$

is well-known to be exactly solvable. Here $\operatorname{sn}(x, m)$ is a Jacobi elliptic function of real elliptic modulus parameter $m(0 \leq m \leq 1)$ with period $4 K(m)$. For simplicity, from now onward, we will not explicitly display the modulus parameter $m$ as an argument of Jacobi elliptic functions [2].

Recently, we have vastly extended [1] the list of known solvable potentials by exploiting two different directions. Firstly, we have shown that the supersymmetric partners of the Lamé potential constitute a wide class of new exactly solvable periodic potentials, which are distinctly different from the Lamé potential of eq. (1) (for $a>1$ ), even though they have the same energy band structure. Secondly, we have shown [1] that the associated Lamé potentials [3]

$$
V(x)=p m \operatorname{sn}^{2} x+q m \frac{\mathrm{cn}^{2} x}{\operatorname{dn}^{2} x}, \quad p \equiv a(a+1), \quad q \equiv b(b+1),
$$

(which constitute a much richer class of periodic potentials) and their supersymmetric partners yield many additional solvable and quasi-exactly solvable (QES) periodic problems provided $a+b$ and/or $a-b$ is an integer. Here, like $\operatorname{sn} x$, the Jacobi elliptic functions $\operatorname{cn} x$ and $\operatorname{dn} x$ also have the same modulus parameter $m$ which, for notational convenience, is not explicitly displayed. Without any loss of generality, we shall always consider associated Lamé potentials with $p \geq q$ [1]. All associated Lamé potentials have a period $2 K(m)$, but the special case $p=q$ has a period $K(m)$.

There are several issues which were not addressed in our previous paper [1], hereafter simply referred to as I. For example, in the case of the Lamé potential (1), it is well known that there are $a(|a+1|)$ bound bands followed by a continuum band when $a$ is a positive (negative) integer $\| \llbracket$. What about the associated Lamé case, especially when both $a$ and $b$ are integers? In particular, for these cases, are there only a finite number of bound bands followed by a continuum band? What happens if $a$ and $b$ are not integers but either $a+b$ or $a-b$ are integers? Furthermore, for the Lamé potential, when $a$ takes half integral values (say $a=n+1 / 2, \quad n=0,1,2, \ldots)$, then $(n+1)$ doublydegenerate solutions are known [3, 4] for the mid-band states (i.e. states with period $8 K$ ). Can one also analytically obtain the mid-band states for the associated Lamé potentials? 
The purpose of this paper is to address the issues raised above. In particular, using certain known theorems about periodic problems in one dimension, we show that even in the associated Lamé case, there are only a finite number of bound bands followed by a continuum band in case both $a$ and $b$ are integers. Further, so long as $a$ and $b$ (and hence $p, q$ ) are unequal integers, then we also show that some of the band gaps (either of period $2 K$ or $4 K$ ) disappear. On the other hand, if $a, b$ are not integers but either $a+b$ or $a-b$ is an integer $(a \neq b)$, then in general there are an infinite number of bands out of which, but for the lowest few, all other band gaps of either period $2 K$ or $4 K$ vanish. Finally, when $a$ is a half integer and $b$ is an integer, we obtain several exact mid-band states (i.e. states of period $8 K)$.

The plan of this paper is as follows. In Sec. II we describe some known theorems about the number of band gaps in a periodic potential in one dimension. Using these theorems, we show that if $a$ and $b$ are integers such that $p>q>0$, then the associated Lamé potential has also only $a$ bound bands followed by a continuum band. Further, if $a-b$ is an even (odd) integer, then there are $b$ doubly degenerate band edges of period $4 K(2 K)$ (i.e. in these cases the corresponding band gaps are zero). Unfortunately, we are unable to obtain energy eigenstates for any of these $2 b$ states. However, we do obtain exact expressions for the remaining $2 a+1$ band edges. In particular, if $a-b$ is an even (odd) integer, then one can obtain the energy eigenvalues and eigenfunctions for the $a+b+1(a-b)$ states

of period $2 K$ and $a-b(a+b+1)$ states of period $4 K$. In Sec. III we discuss the case when both $a, b$ are half integral and such that $p>q>0$. In this case we show that if $a-b$ is an even (odd) integer, one can obtain exact eigenvalues and eigenfunctions for the $b+1 / 2$ doubly degenerate states of period $4 K(2 K)$ (i.e. in these cases, the corresponding band gaps are zero) as well as $a-b$ nondegenerate states of the same period. Unfortunately, in this case one is not able to obtain any eigenstates of period $2 K(4 K)$. In Sec. IV we consider the case when $a$ is half-integral $(a=k+1 / 2)$ and $b$ is an integer $(b=s, \quad s=0,1,2, \ldots, N, \quad k=N-s)$ and show that for every possible value of $s$, one can obtain exact, doubly-degenerate, $k+1$ mid-band states of period $8 K$. Finally, in the last section we summarize the results obtained in this paper and point out some open problems.

\section{II. $a, b$ Integral and Finite Number of Bound Bands}

Consider the Schrödinger equation for the associated Lamé potential specified in eq. (2) for the 
usual case of a particle of mass $1 / 2$ using units with $\hbar=1$ :

$$
-\frac{d^{2} \psi(n)}{d x^{2}}+\left[a(a+1) m \mathrm{sn}^{2}(a)+b(b+1) m \frac{\mathrm{cn}^{2} x}{\operatorname{dn}^{2} x}\right] \psi(x)=E \psi(x) .
$$

Following the procedure described in I, we substitute

$$
\psi(x)=[\operatorname{dn} x]^{-b} y(x),
$$

yielding

$$
y^{\prime \prime}(x)+2 b m \frac{\operatorname{sn} x \operatorname{cn} x}{\operatorname{dn} x} y^{\prime}(x)+\left[\lambda-(a+b)(a+1-b) m \operatorname{sn}^{2} x\right] y(x)=0 .
$$

On further substituting $\operatorname{sn} x \equiv \sin t, y(x) \equiv z(t)$, it is easily shown that $z(t)$ satisfies Ince's equation

$$
(1+A \cos 2 t) z^{\prime \prime}(t)+B \sin 2 t z^{\prime}(t)+(C+D \cos 2 t) z(t)=0
$$

where

$$
\begin{aligned}
A & =\frac{m}{2-m}, \quad B=\frac{(2 b-1) m}{2-m}, \quad C=\frac{\lambda-(a+b)(a+1-b) m}{2-m}, \\
D & =\frac{(a+1-b)(a+b) m}{2-m}, \quad \lambda=E-m b^{2} .
\end{aligned}
$$

Now several exact results for Ince's equation are known in the literature. In particular, it is known that the system satisfying Ince's eq. (6) has at most $j+1$ band gaps of period $\pi[2 \pi]$ in case the polynomial $Q(\mu)\left[Q^{*}(\mu)\right]$ has nonnegative integral roots, the highest of which is $j$ [5]. Here the quadratic polynomials $Q(\mu)$ and $Q^{*}(\mu)$ are given by

$$
Q(\mu)=2 A \mu^{2}-B \mu-\frac{D}{2}, \quad Q^{*}(\mu)=2 A\left(\mu-\frac{1}{2}\right)^{2}-B\left(\mu-\frac{1}{2}\right)-\frac{D}{2} .
$$

On the other hand, if $Q(\mu)\left[Q^{*}(\mu)\right]$ has negative integral roots, the smallest of which is $-j_{0}-1$, then the system satisfying Ince's eq. (6) has at most $j_{0}+1$ band gaps of period $\pi[2 \pi]$. It must be noted here that in the notation of [5], the lowest band gap of period $\pi$ always exists and is from $E=-\infty$ to $E=E_{0}$, when $E_{0}$ denotes the energy of the lower edge of the lowest energy band.

Using these theorems it is easily shown [3] that the Lamé potential (1) has $a(|a+1|)$ bound bands followed by a continuum band in case $a$ is a positive (negative) integer.

Let us now apply these results to the associated Lamé potential (2). We might add here that the period $\pi[2 \pi]$ for Ince's equation corresponds to period $2 K(m)[4 K(m)]$ in the associated Lamé case 
(note $\operatorname{sn} x \equiv \sin t$ ). On using the expressions for A,B,C,D as given in eq. (7) it is easily shown that the roots of $Q(\mu)$ are at

$$
\mu_{1}=\frac{a+b}{2}, \quad \mu_{2}=\frac{b-a-1}{2}
$$

while the roots of $Q^{*}(\mu)$ are at

$$
\mu_{1}^{*}=\frac{a+b+1}{2}, \quad \mu_{2}^{*}=\frac{b-a}{2} .
$$

These roots are integral if and only if $\mathrm{a}+\mathrm{b}$ and/or $\mathrm{a}-\mathrm{b}$ take integer values. In this section we consider the case when both a and b are integral while in the next section we consider the other possibilities.

When both $\mathrm{a}$ and $\mathrm{b}$ take integer values then it follows from eqs. (9) and (10) that either the roots $\mu_{1}$ and $\mu_{2}^{*}$ or the roots $\mu_{2}$ and $\mu_{1}^{*}$ are integral. In particular, it both a and b or odd or even then the roots $\mu_{1}$ and $\mu_{2}^{*}$ are integral while if one of them is odd and the other even then $\mu_{2}$ and $\mu_{1}^{*}$ are integral. Thus in both cases, there are only finite number of band gaps of period $\pi$ and $2 \pi$ ( and hence of periods $2 K$ and $4 K$ for the associated Lamé potential (2)). Hence it follows that when both $a$ and $b$ take integer values, then there are only a finite number of bound bands followed by a continuous band.

In fact, as we show now, these theorems when supplemented with the exact results obtained in I (see Table III of I) tell us quite a lot about the nature of band structure in these cases. We consider the two cases of $a-b$ being odd or even integer separately.

(i) $a-b=$ odd integer:

From eq. (9) it then follows that there are at most $\frac{a-b+1}{2}$ number of band gaps of period $2 K$. An examination of the few explicit cases confirms the fact that there are indeed so many band gaps (and not less) of period $2 K$. This implies that in this case there are only $a-b$ number of nondegenerate states of period $2 K$. Quite remarkably, all these $a-b$ states are QES states. In particular, the solution for these states can be obtained from Table III of I in case $\mathrm{p}=\mathrm{a}(\mathrm{a}+1)$ while $q=[a-(a-b-1)][a-(a-b)]$.

On the other hand, from eq. (10), it follows that in case $a-b$ is an odd integer, then there are at most $\frac{a+b+3}{2}$ number of band gaps of period $4 K$. However, specific examples show that there are in fact only $\frac{a+b+1}{2}$ number of band gaps. This also follows from eq. (10) in case we take $a>0$ but instead of $b$ take $-b-1$ (note that $q$ is invariant under $b \rightarrow-b-1$ ). We see that $\mu_{2}^{*}$ has an integral root at $-\frac{a+b+1}{2}$ and hence there are at most $\frac{a+b+1}{2}$ number of band gaps i.e. $a+b+1$ number of nondegenerate states of period $4 K$. Again all these are QES states, the solution for which is obtained from Table III of I in case $p=a(a+1), q=[a-(a+b)][a-(a+b+1)]$. 
Thus we see that when $a-b$ is an odd integer then there are $\frac{a+b+1}{2}$ band gaps of period $4 K$ but only $\frac{a-b+1}{2}$ band gaps of period $2 K$ and the corresponding band edges are known in principle from Table III of I. However, since the band edge wavefunctions arranged in order of increasing energy are of period $2 K, 4 K, 4 K, 2 K, 2 K, \ldots$, hence it follows that in this case there must also be $b$ band gaps of period $2 K$ which must be of zero width i.e., there must be $b$ doubly degenerate states of period $2 K$. Unfortunately, so far, we have not been able to obtain either the eigenvalues or the eigenfunctions of even one of these states. Thus in this case there are in all $a$ bound bands followed by a continuum band out of which the top $b$ bound bands are a bit unusual in that both of their band edges have period $4 K$ and two degenerate states of period $2 K$ lie inside each of these bound bands.

As an illustration, consider the case of $p=12, q=6$, i.e. $a=3, b=2$. From the above discussion it follows that there must be 1 QES band edge of period $2 K$ which is the ground state. Using Table III of I, it is easily seen that the eigenvalue and the eigenfunction of this state is

$$
\psi_{0}=\mathrm{dn}^{3} x, \quad E_{0}=9 m
$$

In addition, there must be 6 nondegenerate QES band edges of period $4 K$ and the eigenvalues and eigenfunctions for these six states are easily obtained. In particular, it is easily shown that three of the eigenstates have the form

$$
\psi_{1,6,9}=\frac{\operatorname{cn} x}{\operatorname{dn}^{2} x}\left[A+B \operatorname{sn}^{2} x+D \operatorname{sn}^{4} x\right]
$$

and the corresponding three eigenvalues $E_{1,6,9}$ satisfy the cubic equation

$$
\lambda^{3}-4(8-m) \lambda^{2}+48(4+m) \lambda-576 m=0, \quad E=\lambda+1+4 m
$$

The other three eigenstates have the form

$$
\psi_{2,5,10}=\frac{\operatorname{sn} x}{\operatorname{dn}^{2} x}\left[A+B \operatorname{sn}^{2} x+D \operatorname{sn}^{4} x\right]
$$

and the corresponding three eigenvalues $E_{2,5,10}$ satisfy the cubic equation

$$
\lambda^{3}-8(4+m) \lambda^{2}+48(4+7 m) \lambda-576 m(3+m)=0, \quad E=\lambda+1+m
$$

In view of the oscillation theorem, it is then clear that there must be a pair of doubly degenerate states $\left(\psi_{3,4}\right)$ and $\left(\psi_{7,8}\right)$ of period $2 K$ whose energy must go to $4(14)$ and $16(17)$ respectively as $m \rightarrow 0(1)$. Thus whereas four states $\psi_{2,3,4,5}$ must merge at $E=14$ as $m \rightarrow 1$ the other four states $\psi_{6,7,8,9}$ must 
merge at $E=17$ as $m \rightarrow 1$. Thus, as shown in Fig. 1, in this case there are three bound bands followed by a continuum band. The two upper-most bound bands have both of their band edges of period $4 K$ and in between are the pair of doubly degenerate states $\psi_{3,4}$ and $\psi_{7,8}$ of period $2 K$ whose energy eigenvalues and eigenfunctions are not known analytically. We have therefore computed these energy eigenvalues numerically and these are shown by the dotted lines in the figure.

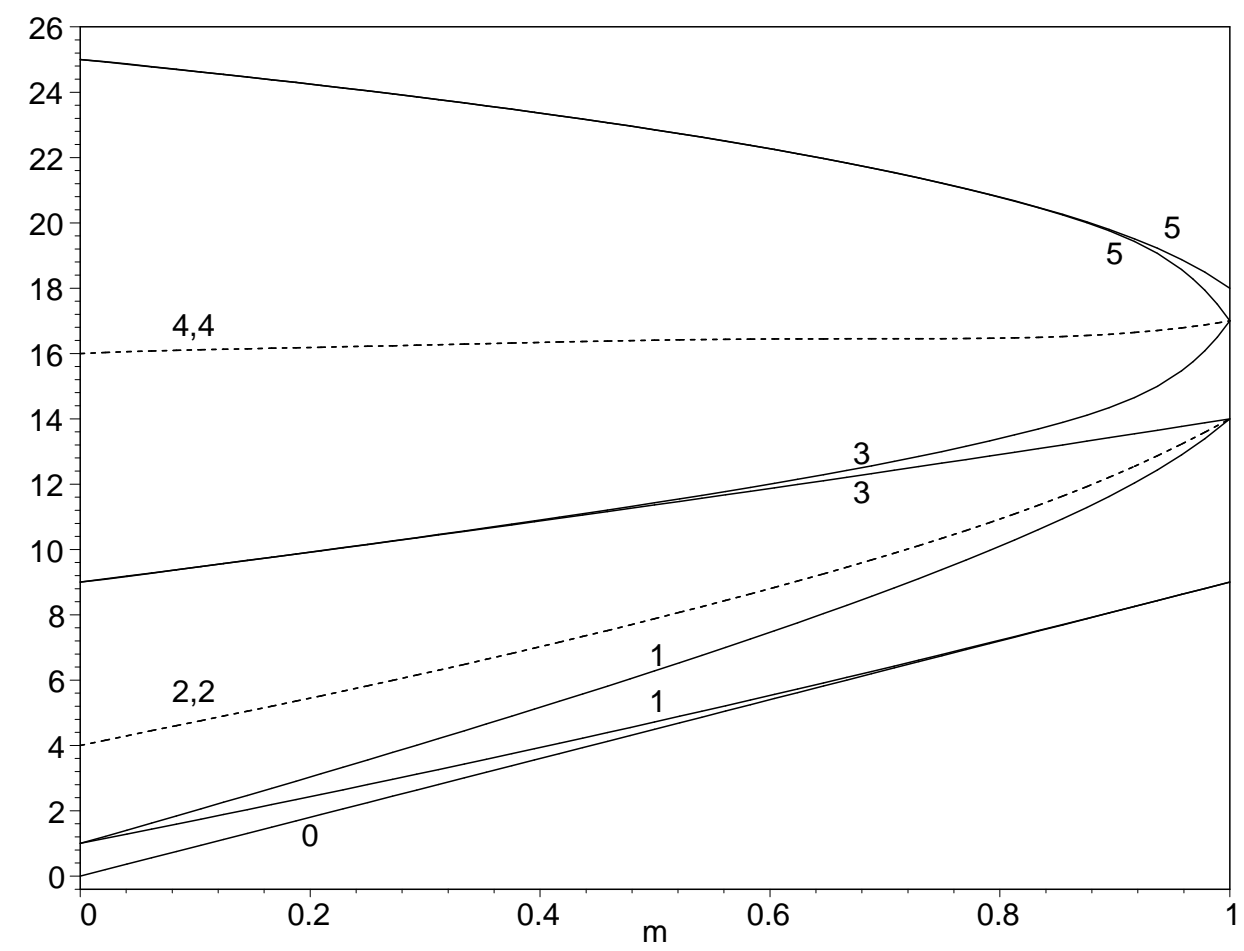

Figure 1: Band edge energies for the associated Lamé potential $(12,6)$ as a function of the elliptic modulus parameter $m$. The band edges are labelled by the number of wave function nodes in the interval $2 K(\mathrm{~m})$. Note that the band gap between the two states with 2 nodes as well as with four nodes is zero, that is $E_{3}=E_{4}$ and $E_{7}=E_{8}$. The energy eigenvalues of these degenerate states have been calculated numerically and is shown by dotted lines.

As another illustration, consider the case of $p=6, q=2$, i.e. $a=2, b=1$. From the above discussion it follows that in this case there must be 1 QES band edge of period $2 K$ and 4 QES band edges of period $4 K$ and interestingly enough the eigenvalues and eigenfunctions for these five states were already given in the Table IV of I. Further, as stated in I, two states of period $2 K$ must also be present but so far we are unable to obtain these states analytically. However, what was not clear at that time was that the two states of period $2 K$ must be degenerate and whose energy goes to $4(7)$ as 
$m \rightarrow 0$ (1) so that as $m \rightarrow 1$, four of the states $\psi_{2,3,4,5}$ must merge at $E=7$. Besides, it was also not clear then that in this case there are only two bound bands followed by a continuum band and that the upper band is a bit unusual in that both of its band edges are of period $4 K$ and inside the band there are two degenerate states of period $2 K$.

(ii) $a-b=$ even integer:

From eq. (9), it follows that in this case there are at most $\frac{a+b+2}{2}$ band gaps of period $2 K$. Explicit examples confirm that this is indeed so. Hence, in this case one has $a+b+1$ number of nondegenerate states. Quite remarkably, all these are QES states for which solution can be obtained from Table III of I when $p=a(a+1), q=[a-(a+b)][a-(a+b+1)]$.

On the other hand, from eq. (10), it follows that there are at most $\frac{a-b}{2}$ band gaps of period $4 K$ and hence $a-b$ number of nondegenerate states of period $4 \mathrm{~K}$. Specific examples confirm the expectation. These are all QES states which can be obtained from Table III of I in case $p=a(a+1), q=$ $[a-(a-b)][a-(a-b-1)]$.

Thus when $a-b$ is an even integer (with both $a, b$ integer), then there are $\frac{a+b+2}{2}$ band gaps of period $2 K$ but only $\frac{a-b}{2}$ band gaps of period $4 K$ and in principle the corresponding band edges are all analytically known from Table III of I. In view of the fact that the band edge wave functions in increasing order of energy are of period $2 K, 4 K, 4 K, 2 K, 2 K, \ldots$, it then follows that in this case too there must be $b$ zero band gaps of period $4 K$ i.e. there must be $b$ doubly degenerate states of period $4 K$. Unfortunately, so far, we have not been able to obtain analytic solution for even one of these (2b) states. Thus in this case also there are in all $a$ bound bands followed by a continuum band out of which the top $b$ bound bands are again bit unusual in that both of their band edges are of period $2 K$ and two degenerate states of period $4 K$ lie inside each of these bound bands.

As an illustration, consider the case of $p=12, q=2$ i.e. $a=3, b=1$. As described in I, this potential is oscillatory for $m<\frac{5}{6}$, but has interesting structure coming from secondary extrema for $m>\frac{5}{6}$. From the above discussion it follows that the $(12,2)$ potential must have 2 nondegenerate QES states of period $4 K$. Using Table III of I, it is easily seen that the eigenvalues and the eigenfunctions of these states is given by

$$
\begin{aligned}
& \psi_{1}=\operatorname{cn} x \operatorname{dn}^{2} x, \quad E_{1}=1+4 m, \\
& \psi_{2}=\operatorname{sn} x \operatorname{dn}^{2} x, \quad E_{2}=1+9 m .
\end{aligned}
$$

In addition there must be 5 nondegenerate states of period $2 K$ whose eigenvalues and eigenfunctions 
are easily obtained from Table III of I. In particular, the eigenvalues and the eigenfunctions of two of the states are

$$
\psi_{8,3}=\frac{\operatorname{sn} x \operatorname{cn} x}{\operatorname{dn} x}\left[5 m \mathrm{sn}^{2} x-3-m \pm \delta_{7}\right], \quad E_{8,3}=10+2 m \pm 2 \delta_{7},
$$

where $\delta_{7}=\sqrt{9-9 m+m^{2}}$. On the other hand, the remaining three eigenstates have the form

$$
\psi_{0,4,7}=\frac{1}{\operatorname{dn} x}\left[A+B \operatorname{sn}^{2} x+D \operatorname{sn}^{4} x\right],
$$

and the three corresponding eigenvalues $E_{0,4,7}$ satisfy the cubic equation (see eqs. (39) and (40) of I)

$$
\lambda^{3}-4(2 m+5) \lambda^{2}+16(4+11 m) \lambda-192 m(2+m)=0, \quad E=\lambda+m .
$$

In view of the oscillation theorem, it is then clear that there must also be two degenerate states $\left(\psi_{5,6}\right)$ of period $4 K$ whose energy tends to $9(13)$ as $m \rightarrow 0(1)$ so that the four states $\psi_{4,5,6,7}$ must merge at $\mathrm{E}=13$ as $m \rightarrow 1$. Thus, as shown in Fig. 2, in this case, there are three bound bands followed by a continuum band. Further, the upper-most bound band has both of its band edges of period $2 K$ and in between are the two degenerate states of period $4 K$ whose energy eigenvalue and eigenfunctions are not known analytically. We have computed the degenerate energy eigenvalue numerically and it is shown by a dotted line in Fig. 2.

What happens if $a=b$ with both being integer? In this case the associated Lamé potential has period $K(m)$ rather than $2 K(m)$ and hence the results from Ince's equation are not directly applicable. However, as has been shown in I for $a=b=1,2$ ( and also explicitly verified for $a=b=3,4$ ), in this case there are $a$ bound bands followed by a continuum band and $2 a+1$ band edges all of which are in principle explicitly known from Table III of I. Further, in this case, there are no band gaps of zero width.

\section{III. $a, b$ Half Integral and Infinite Number of Band Gaps of Zero Width}

Let us now specialize to the case when both $a$ and $b$ are half-integral such that $p>q>0$. From eqs. (9) and (10) it is clear that in this case either the roots $\mu_{1}$ and $\mu_{2}$ or $\mu_{1}^{*}$ and $\mu_{2}^{*}$ are integral but not the both. In particular, if $a-b$ is an odd integer, then both the roots $\mu_{1}$ and $\mu_{2}$ are integral while $\mu_{1}^{*}$ and $\mu_{2}^{*}$ are not integral while if $a-b$ is an even integer, then the roots $\mu_{1}^{*}$ and $\mu_{2}^{*}$ are integral while $\mu_{1}$ and $\mu_{2}$ are not integral. Thus it follows that unlike the integral $a, b$ case, here one will in general have infinite number of bands but only finite number of band gaps of either period $2 K$ or $4 K$ depending on whether $a-b$ is an odd or an even integer. As before, let us discuss the two cases separately. 


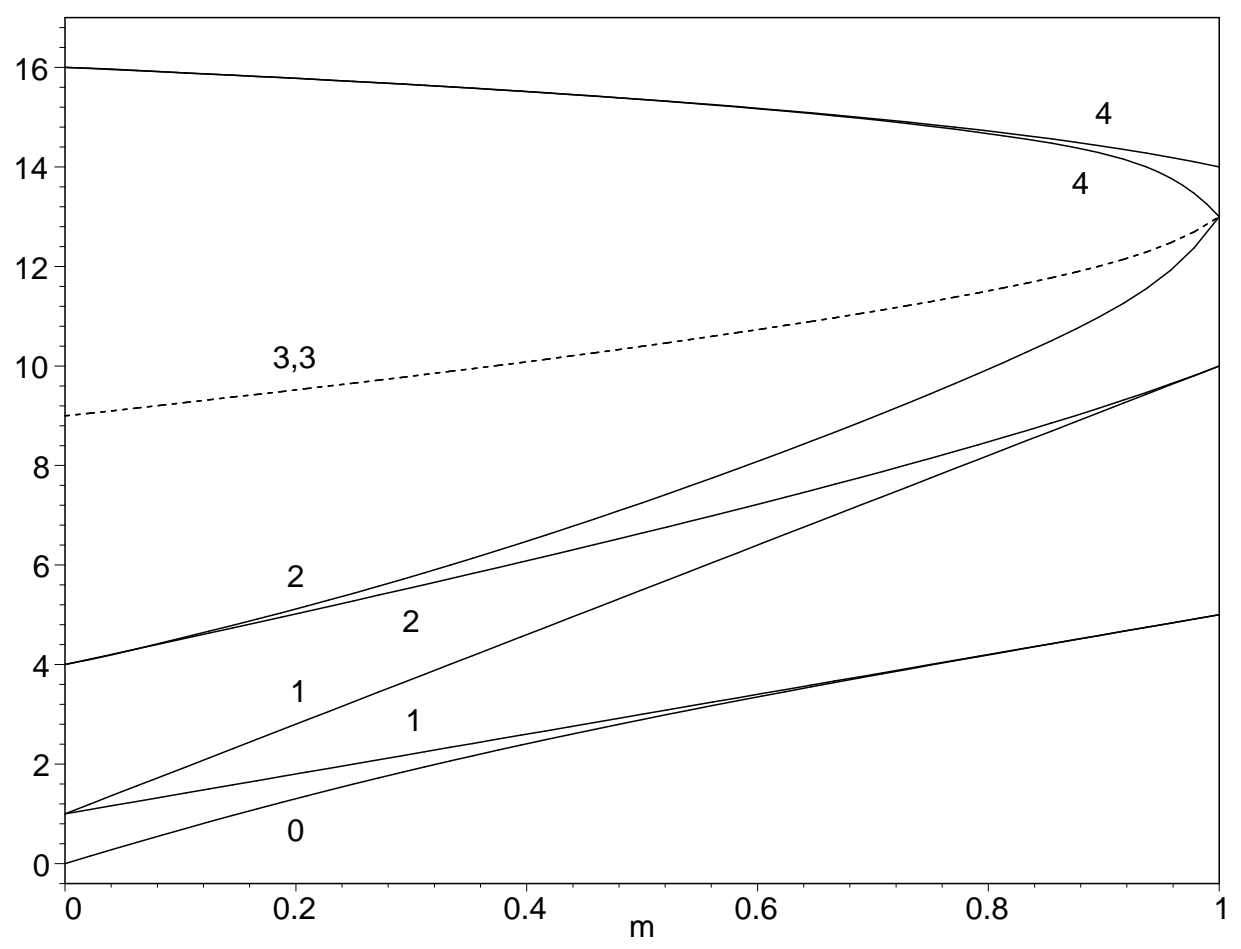

Figure 2: Band edge energies for the associated Lamé potential $(12,2)$ as a function of the elliptic modulus parameter $m$. The band edges are labelled by the number of wave function nodes in the interval $2 K(m)$. Note that the band gap between the two states with 3 nodes is zero. The energy eigenvalue $E_{5}=E_{6}$ of these degenerate states has been calculated numerically and is shown by a dotted line.

(i) $a-b=$ odd integer:

From eqs. (9) and (10) it follows that in this case both $\mu_{1}$ and $\mu_{2}$ are integral while $\mu_{1}^{*}$ and $\mu_{2}^{*}$ are half integral. Thus in this case, there will be infinite number of band gaps of period $4 \mathrm{~K}$ but at most $\frac{a+b+2}{2}$ band gaps of period $2 K$. However, a study of several explicit examples show that there are in fact only $\frac{a-b+1}{2}$ number of band gaps of period $2 K$ and hence there are only $a-b$ number of nondegenerate states of period $2 K$ and all these are QES states. However, using Table III of I it is easily shown that in this case there are in fact $a+b+1$ QES states of period $2 K$. This then implies that the remaining $2 b+1$ QES states must correspond to doubly degenerate eigenstates of period $2 K$ i.e. in this case there are $b+\frac{1}{2}$ doubly degenerate QES states of period $2 K$, each of which is lying inside a band. It is interesting to note that whereas when both a and $\mathrm{b}$ are integers, then one does not have analytic expression for even one doubly degenerate state, when both a,b are half integral, energy 
eigenvalues and eigenfunctions are known in principle from Table III of I for $b+\frac{1}{2}$ doubly degenerate QES states.

Summarizing, when $\mathrm{a}-\mathrm{b}$ is an odd integer, then there are infinite number of bands out of which except for the lowest $a-b$ bands, the rest are rather unusual in that both of their band edges have period $4 K$ and two degenerate states of period $2 K$ reside inside each of these bands.

As an illustration, consider the case of $p=\frac{15}{4}, q=3 / 4$ i.e. $a=3 / 2, b=1 / 2$. From the above discussion, it follows that in this case one must have one nondegenerate and one doubly degenerate QES state of period $2 K$. Using Table III of I it is easily seen that the eigenvalue and the eigenfunction for the nondegenerate state is given by

$$
\psi_{0}=\mathrm{dn}^{3 / 2} x, \quad E_{0}=\frac{9 m}{4},
$$

while the energy eigenvalue and the corresponding two degenerate eigenfunctions are given by

$$
\psi_{3}=\frac{\operatorname{sn} x \operatorname{cn} x}{\operatorname{dn}^{1 / 2} x}, \psi_{4}=\frac{\left[2 \operatorname{sn}^{2} x-1\right]}{\operatorname{dn}^{1 / 2} x}, \quad E_{3,4}=4+\frac{m}{4} .
$$

Note that in this case, out of the infinite number of bands, except for the lowest band, all other bands have both of their band edges of period $4 \mathrm{~K}$.

Another case, already discussed in I (see Table VII) is when $p=\frac{63}{4}, q=3 / 4$ i.e. $a=7 / 2, b=1 / 2$. As shown there, in this case one has three nondegenerate QES states of period $2 K$ and one doubly degenerate level of the same period. Further, in this case, but for the lowest three bands, all other bands have both of their band edges of period $4 \mathrm{~K}$.

(ii) $a-b=$ even integer:

From eqs. (9) and (10) it follows that in this case both $\mu_{1}^{*}$ and $\mu_{2}^{*}$ are integral while $\mu_{1}$ and $\mu_{2}$ are half integral. Hence, in this case there will be infinite number of band gaps of period $2 K$ but at most $\frac{a+b+1}{2}$ number of band gaps of period $4 \mathrm{~K}$. However, a study of several explicit examples reveals that in this case there are only $\frac{a-b}{2}$ number of band gaps and hence $\mathrm{a}$ - b number of nondegenerate QES states of period $4 \mathrm{~K}$. However, using Table III of I it is easily shown that in this case there are in fact $\mathrm{a}+\mathrm{b}+1$ number of $\mathrm{QES}$ states of period $4 K$. This implies that in addition to the a - $\mathrm{b}$ nondegenerate QES states, there must also be $2 \mathrm{~b}+1$ QES degenerate states of period $4 K$ i.e. in this case also there are $b+1 / 2$ doubly degenerate QES states of period $4 K$, each of which is lying inside a band.

Thus, in this case too, there are an infinite number of bands out of which except for the lowest $\mathrm{a}-\mathrm{b}$ bands, the rest are rather unusual in that both of their band edges are of period $2 K$ and two degenerate states of period $4 K$ reside inside each of these bands. 
As an illustration, consider the case of $p=\frac{35}{4}, q=3 / 4$ i.e. $a=5 / 2, b=1 / 2$. In view of the above discussion, we expect two nondegenerate and one doubly degenerate QES states of period $4 K$. Using Table III of I it is easily shown that the energy eigenstates for the two nondegenerate states are

$$
\begin{aligned}
& \psi_{1}=\operatorname{cn} x \operatorname{dn}^{3 / 2} x, E_{1}=1+\frac{9 m}{4}, \\
& \psi_{2}=\operatorname{sn} x \operatorname{dn}^{3 / 2} x, E_{2}=1+\frac{25 m}{4},
\end{aligned}
$$

while for the two degenerate states one has

$$
\psi_{5}=\frac{\operatorname{cn} x\left(4 \operatorname{sn}^{2} x-1\right)}{\operatorname{dn}^{1 / 2} x}, \psi_{6}=\frac{\operatorname{sn} x\left(4 \operatorname{sn}^{2} x-3\right)}{\operatorname{dn}^{1 / 2} x}, E_{5,6}=9+\frac{m}{4} .
$$

Thus, in this case, (out of the infinite number of bands) except for the two lowest bands, all other bands are a bit unusual in that both of their band edge eigenfunctions are of period $2 K$.

We thus have seen that for half integral $a, b$ with $a>b$, one has $a-b$ nondegenerate and $b+\frac{1}{2}$ doubly degenerate QES states of period $2 K$ or $4 K$ depending on whether $a-b$ is an odd or an even integer. Thus for $b=1 / 2,3 / 2, \ldots(a>b)$, we expect $1,2, \ldots$ doubly degenerate QES states. We now show that the energy eigenvalues of these doubly degenerate states can be easily obtained analytically in case $b=1 / 2$ or $3 / 2$ and $a$ being arbitrary half integer (with $a>b$ ). In particular, if we start with the ansatz

$$
y_{1}(x)=\sum_{k=0}^{N+1} A_{k} \operatorname{sn}^{2 k} x,
$$

then on substituting it in the associated Lamé eq. (5) and equating coefficient of terms with $\operatorname{sn}^{2 N+4} x$ and $\operatorname{sn}^{2 N+2} x$ we find that in case $a=2 N+3 / 2, b=1 / 2$ then the corresponding energy eigenvalue is

$$
E=(2 N+2)^{2}+m / 4, N=0,1,2, \ldots
$$

In fact $E$ as given by eq. (27) is also the energy eigenvalue in case we start with the ansatz

$$
y_{2}(x)=\sum_{k=0}^{N} A_{k} \operatorname{sn}^{2 k+1} x \operatorname{cn} x,
$$

and substitute it in eq. (5). As expected, the results given in eq. (22) agree with these given above in case $\mathrm{N}=0$ while for $\mathrm{N}=1$ our results agree with those given in Table VII of I.

Similarly, it is easily shown that in case $a=2 N+5 / 2, b=1 / 2$ then the degenerate eigenvalue and the corresponding eigenfunctions are

$$
E=(2 N+3)^{2}+m / 4, N=0,1,2, \ldots
$$




$$
y_{1}(x)=\sum_{k=0}^{N+1} A_{k} \operatorname{sn}^{2 k+1} x, \quad y_{2}(x)=\sum_{k=0}^{N+1} B_{k} \operatorname{cn} x \operatorname{sn}^{2 k} x .
$$

For the special case of $N=0$, our results agree with those given in eq. (25).

On the other hand, for $b=3 / 2$ and $a=2 N+5 / 2$, the two degenerate energy eigenvalues are

$$
E=4 N^{2}+12 N+10+\frac{5 m}{4} \pm \sqrt{(4 N+6)^{2}-(4 N+6)^{2} m+m^{2}}, N=0,1,2, \ldots,
$$

while the corresponding eigenfunctions are of the form

$$
y_{1}(x)=\sum_{k=0}^{N+2} A_{k} \operatorname{sn}^{2 k} x, \quad y_{2}(x)=\sum_{k=0}^{N+1} B_{k} \operatorname{cn} x \operatorname{sn}^{2 k+1} x .
$$

However, for $b=3 / 2$ and $a=2 N+7 / 2$, the two degenerate energy eigenvalues are

$$
E=4 N^{2}+16 N+17+\frac{5 m}{4} \pm \sqrt{16(N+2)^{2}-16(N+2)^{2} m+m^{2}}, \quad N=0,1,2, \ldots,
$$

while the corresponding degenerate eigenfunctions are of the form

$$
y_{1}(x)=\sum_{k=0}^{N+2} A_{k} \operatorname{sn} x^{2 k+1}, \quad y_{2}(x)=\sum_{k=0}^{N+2} B_{k} \operatorname{cn} x \operatorname{sn}^{2 k} x .
$$

It is worth adding that in case $b=-1 / 2(a>b)$, one obtains $a+\frac{1}{2}$ nondegenerate QES states of period $2 K(4 K)$ depending on if $a+\frac{1}{2}$ is an odd or an even integer. Note that when $b=-1 / 2$ then $q=-1 / 4$ while $p>0$. As an illustration, consider $a=5 / 2, b=-1 / 2$ i.e. $p=35 / 4, q=-1 / 4$. In this case we have 3 QES states of period $2 K$ whose energy eigenvalues and eigenfunctions are easily obtained from Table III of I when $q=(a-2)(a-3)$ and of course $p=a(a+1)$.

Finally, let us discuss the case when $a, b$ are neither integral nor half integral but are such that either $a+b$ or $a-b$ is an integer. It is easily seen from eqs. (9) and (10) that if either $a+b=2 N$ or $a-b=2 N+1$ then there are at most $N+1$ band gaps of period $2 K$ while if $a-b=2 N$ or $a+b=2 N-1$ then there are at most $N$ band gaps of period $4 K$. Further, from Table III of I we find that when either $a+b=2 N$ or $a-b=2 N+1$ then there are precisely $2 N+1$ QES states of period $2 K$ while if $a+b=2 N-1$ or $a-b=2 N$ then there are precisely $2 N$ QES states of period $4 K$ and all these are nondegenerate states.

\section{Mid-Band States}

For the Lamé potential, the majority of results are for integral $a$ (and hence $p$ ). However, for half-integral values of $a$, analytic expressions for $a+\frac{1}{2}$ mid-band states (of period $8 K$ ) have been 
obtained 《4. In particular, it is known that if $a=2 N+\frac{1}{2}$, there are $2 N+1$ mid-band states of the form $(N=0,1,2, \ldots)$

$$
\psi(x)=\sqrt{\operatorname{dn} x+\operatorname{cn} x} u(x)
$$

where

$$
u(x)=\sum_{k=0}^{N} A_{k} \operatorname{sn}^{2 k} x+\sum_{k=0}^{N-1} B_{k} \operatorname{cn} x \operatorname{dn} x \operatorname{sn}^{2 k} x,
$$

while if $a=2 N+\frac{3}{2}$, then there are $2 N+2$ states of the form $(35)$ but where $(N=0,1,2, \ldots)$

$$
u(x)=\operatorname{dn} x \sum_{k=0}^{N} A_{k} \operatorname{sn}^{2 k} x+\operatorname{cn} x \sum_{k=0}^{N} B_{k} \operatorname{sn}^{2 k} x .
$$

Further, since the Lamé equation is invariant under $x \rightarrow x+2 K(m)$, it follows that in each case one obtains another solution with the same energy by changing $\operatorname{cn} x$ to $-\operatorname{cn} x$.

In this section we show that the associated Lamé potential (2) also has a similar form of midband solutions (of period $8 K$ ) in case $a$ is half integral while $b$ takes integral values. In particular, if $a=k+\frac{1}{2}, b=s$ while $k=2 N$ with $N, k, s$ being nonnegative integers, then for a given $k$, one obtains $k+1$ doubly degenerate solutions for every possible (non-negative integral) value of $s$. Further, in this case too, all solutions are also doubly degenerate since the associated Lamé equation is invariant under $\operatorname{cn} x \rightarrow-\operatorname{cn} x$.

We begin by substituting the ansatz

$$
y(x)=\sqrt{\operatorname{dn} x+\operatorname{cn} x} z(x)
$$

into eq. (5). We find that $z(x)$ satisfies the equation

$$
\begin{aligned}
& \operatorname{sn} x \operatorname{dn} x z^{\prime \prime}(x)+\left[2 b m \operatorname{sn}^{2} x \operatorname{cn} x-\operatorname{dn} x+\operatorname{cn} x \operatorname{dn}^{2} x\right] z^{\prime}(x) \\
& +\left[\lambda_{1} \operatorname{sn} x \operatorname{dn} x-r m \operatorname{sn}^{3} x \operatorname{dn} x-b m \operatorname{cn} x \operatorname{sn} x+b m \operatorname{cn}^{2} x \operatorname{sn} x \operatorname{dn} x\right] z(x)=0,
\end{aligned}
$$

where

$$
\lambda_{1}=\lambda-\frac{1+m}{4}, r=(a+1-b)(a+b)-3 / 4 .
$$

Not surprisingly, $z(x)=$ constant is a solution with energy $E=1+\frac{m}{4}$ (note $E=\lambda+m b^{2}$ ) provided $b=0$ and $a=1 / 2$ i.e. $p=3 / 4, q=0$. Following the discussion given above, we try the ansatz (37) with $N=0$, i.e.

$$
z(x)=A \operatorname{dn} x+B \operatorname{cn} x,
$$


in eq. (39). It is easily shown that there are two possible solutions in this case

(i) $b=0, a=3 / 2$ :

$$
\psi(x)=\left[\operatorname{dn} x-\left(1-m \pm \sqrt{1-m+m^{2}}\right) \operatorname{cn} x\right] \sqrt{\operatorname{dn} x+\operatorname{cn} x}, E=\frac{5}{4}(1+m) \pm \sqrt{1-m+m^{2}} .
$$

(ii) $b=1, a=1 / 2$ :

$$
\psi(x)=\left[1-\frac{2 \operatorname{cn} x}{\operatorname{dn} x}\right] \sqrt{\operatorname{dn} x+\operatorname{cn} x}, E=\frac{9+m}{4} .
$$

Several comments are in order at this stage.

1. On making use of the fact that as $m \rightarrow 0, \operatorname{dn} x \rightarrow 1$ while $\operatorname{cn} x \rightarrow \cos x$ it is easily shown that in case $b=0, a=3 / 2$, the two solutions go over to $\cos \frac{x}{2}$ and $\cos \frac{3 x}{2}$ with energies $\frac{1}{4}$ and $9 / 4$ respectively. On the other hand, as $m \rightarrow 1$, the two states go over to the ground and excited states of the potential $V=\frac{15}{4} \tanh ^{2} x$ with eigenvalues $\frac{3}{2}$ and $7 / 2$ respectively.

2. On the other hand, as $m \rightarrow 0$ the solution with $b=1$ and $a=1 / 2$ goes over to $\cos \frac{3 x}{2}$ with energy $\frac{9}{4}$ while as $m \rightarrow 1$, it goes over to the ground state of the potential $V=\frac{3}{4} \tanh ^{2} x+2$ with energy eigenvalue $\frac{5}{2}$.

3. Degenerate solutions are obtained in all these cases by changing $\mathrm{cn} x$ to $-\mathrm{cn} x$, and as $m \rightarrow 0$ these go over to $\sin \frac{x}{2}$ or $\sin \frac{3 x}{2}$ as the case may be.

One can now immediately generalize to the general ansatz (37) and show that for a given $N$, if $a=k+1 / 2$ and $b=s$ with $k=2 N+1-s(N=0,1,2, \ldots)$ then $k+1$ doubly degenerate eigenvalues and eigenfunctions can be obtained for every possible nonnegative $s$. For example, let us consider the ansatz (37) with $N=1$, i.e.

$$
z(x)=\operatorname{dn} x\left[A_{0}+A_{1} \operatorname{sn}^{2} x\right]+\operatorname{cn} x\left[B_{0}+B_{1} \operatorname{sn}^{2} x\right] .
$$

After substituting this ansatz in eq. (39) and performing lengthy algebraic manipulations, it is easily shown that there are four possible solutions in this case.

(i) $b=0, a=7 / 2$ :

As is well known 迎, in this case $\lambda_{1}=E-\frac{1+m}{4}$ satisfies a quartic equation

$$
\lambda_{1}^{4}-20(1+m) \lambda_{1}^{3}+18\left(6+19 m+6 m^{2}\right) \lambda_{1}^{2}-36\left(4+39 m+39 m^{2}+4 m^{3}\right) \lambda_{1}+135 m\left(8+23 m+8 m^{2}\right)=0,
$$


all of whose roots are real and that as $m \rightarrow 0$ the solutions go over to $\cos \frac{x}{2}, \cos \frac{3 x}{2}, \cos \frac{5 x}{2}, \cos \frac{7 x}{2}$ with energies $\frac{1}{4}, \frac{9}{4}, \frac{25}{4}$ and $\frac{49}{4}$ respectively.

(ii) $b=1, a=5 / 2$ :

In this case $\lambda_{1}$ can be shown to satisfy the cubic equation (note $E=\lambda_{1}+\frac{1+m}{4}+m b^{2}$ )

$$
\lambda_{1}^{3}-(5 m+14) \lambda_{1}^{2}+\left(24+88 m-m^{2}\right) \lambda_{1}+5 m^{3}-98 m^{2}-96 m=0,
$$

whose all three roots are real for any $m(0 \leq m \leq 1)$. As $m \rightarrow 0$, we find that the solutions go over to $\cos \frac{x}{2}, \cos \frac{3 x}{3}, \cos \frac{7 x}{2}$ with energies $1 / 4,9 / 4,49 / 4$ respectively.

(iii) $b=2, a=3 / 2$ :

In this case there are two solutions with the corresponding energies being

$$
E=\frac{29+5 m}{4} \pm \sqrt{25-25 m+m^{2}}
$$

Note that as $m \rightarrow 0$ the two energies go over to $\frac{9}{4}$ and $\frac{49}{4}$ and the corresponding solutions go over to $\cos \frac{3 x}{2}$ and $\cos \frac{7 x}{2}$ respectively.

(iv) $b=3, a=1 / 2$ :

In this case there is only one solution given by

$$
\psi=\left(\operatorname{dn} x\left[1-\frac{(4-m)}{3} \operatorname{sn}^{2} x\right]-\frac{4}{3} \operatorname{cn} x\left[1-(2-m) \operatorname{sn}^{2} x\right]\right) \sqrt{\operatorname{dn} x+\operatorname{cn} x}, E=\frac{49+m}{4} .
$$

It is easily checked that as $m \rightarrow 0$ the solution goes over to $\cos \frac{7 x}{2}$ and the corresponding energy is $\frac{49}{4}$.

On the other hand if $a=k+\frac{1}{2}, b=s$ and $k=2 N-s(N=0,1,2, \ldots)$ then we start with the ansatz (36) and obtain $k+1$ (doubly degenerate) eigenvalues and eigenfunctions for every possible nonnegative $s$. For $N=0$, the only possibility is of course $a=1 / 2, b=0$ and in this case the solution is already well known [4]. For $N=1$ we start with the ansatz,

$$
z(x)=A_{0}+A_{1} \operatorname{sn}^{2} x+B_{0} \operatorname{cn} x \operatorname{dn} x
$$

On substituting this ansatz in eq. (39), after lengthy but straightforward algebraic manipulations, we find the following three solutions:

(i) $b=0, a=5 / 2$ :

As is well known 沟, in this case $\lambda_{1}=E-\frac{1+m}{4}$ satisfies a cubic equation

$$
\lambda_{1}^{3}-8(1+m) \lambda_{1}^{2}+4\left(3+13 m+3 m^{2}\right) \lambda_{1}-48 m(1+m)=0,
$$


all of whose roots are real and as $m \rightarrow 0$, the solutions go over to $\cos \frac{x}{2}, \cos \frac{3 x}{2}, \cos \frac{5 x}{2}$ with energies $\frac{1}{4}, \frac{9}{4}$ and $\frac{25}{4}$ respectively.

(ii) $b=1, a=3 / 2$ :

In this case there are two solutions with the corresponding energies being

$$
E=\frac{13+5 m}{4} \pm \sqrt{9-9 m+m^{2}}
$$

Note that as $m \rightarrow 0$ the energies go over to $\frac{1}{4}$ and $\frac{25}{4}$ while the corresponding solutions go over to $\cos \frac{x}{2}$ and $\cos \frac{5 x}{2}$ respectively.

(iii) $b=2, a=1 / 2$ :

In this case there is only one solution

$$
\psi=\sqrt{\operatorname{dn} x+\operatorname{cn} x}\left[1-\frac{(4-m)}{3} \operatorname{sn}^{2} x-\frac{2}{3} \operatorname{cn} x \operatorname{dn} x\right], E=\frac{25+m}{4},
$$

As $m \rightarrow 0$ the solution goes over to $\cos \frac{5 x}{2}$ with energy $25 / 4$.

Before closing this section it is worth pointing out that the mid-band states have already been obtained in I in case $a=b=N+1 / 2,(N=0,1,2, \ldots)$. In particular, it may be noted that when $a=b$, then the associated Lamé potential has period $K$ rather than $2 K$ and hence the band edges will be of period $K$ and $2 K$ while the mid-band states will be of period $4 K$. Now if one looks at the Table III of I then one notices that if $a=b=N+1 / 2$, then there are $N+1$ doubly degenerate QES states of period $4 K$ which are obtained in principle from the Table III of I in case $a=N+1 / 2$ and $q=[a-(N+1)][a-(N+2)]$. For example, for $N=0$, i.e. for $p=q=3 / 4$, the doubly degenerate mid-band states are

$$
\psi_{1}=\frac{\operatorname{cn} x}{\sqrt{\operatorname{dn} x}}, \psi_{2}=\frac{\operatorname{sn} x}{\sqrt{\operatorname{dn} x}}, E=1+\frac{m}{4} .
$$

On the other hand, for $N=1$ the pair of doubly degenerate mid-band states are

$$
\begin{gathered}
\psi_{1}=\frac{\operatorname{cn} x\left(4-m-2 \operatorname{sn}^{2} x\right)}{\operatorname{dn}^{3 / 2} x}, \psi_{2}=\frac{\operatorname{sn} x\left(4-2 \operatorname{sn}^{2} x\right)}{\operatorname{dn}^{3 / 2} x}, E=1+\frac{9 m}{4}, \\
\psi_{1}=\frac{\operatorname{cn} x \operatorname{sn}^{2} x}{\operatorname{dn}^{3 / 2} x}, \psi_{2}=\frac{\operatorname{sn} x\left(2 \operatorname{sn}^{2} x-1\right)}{\operatorname{dn}^{3 / 2} x}, E=9+\frac{m}{4} .
\end{gathered}
$$

\section{Conclusion and Open Problems:}

In this paper we have clarified several issues regarding the associated Lamé potential. In particular, we have shown that when both $a, b$ are integers then just like Lamé, the associated Lamé potential is 
also a finite band problem. The only difference from the Lamé case arises when $a \neq b$ - in that case one has some bands with both band edges of the same period. We have also seen that when both $a, b$ take half integral but unequal values then one has a genuine QES problem and band edges of either period $2 K$ or $4 K$ are known, but not both. We have also shown that in this case, but for the few low lying bands, all other bands have both of their band edges of the same period ( $2 K$ or $4 K$ ). Finally, when $a$ is a half integer and $b$ is an integer, we can obtain several mid-band states.

It would be nice if one could (i) say something concrete about the band structure when $a, b$ are neither integral or half integral; (ii) derive dispersion relations for at least some of the finite band associated Lamé problems; (iii) obtain the band edges of the associated Lamé problem algebraically, analogous to the Lamé potential. We hope to address some of these issues in the near future.

\section{References}

[1] A. Khare and U. Sukhatme, J. Math. Phys. 40 (1999) 5473; hereafter, we will refer to this paper as I .

[2] For the properties of Jacobi elliptic functions, see, for example, I. S. Gradshteyn and I. M. Ryzhik, Table of Integrals, Series and Products (Academic Press, 1980). The modulus parameter $m$ is often called $k^{2}$ in the mathematics literature. The related complementary quantity $(1-m)$ is often called $k^{\prime 2}$.

[3] W. Magnus and S. Winkler, Hill's Equation (Wiley, New York, 1966).

[4] F. M. Arscott, Periodic Differential Equations (Pergamon, Oxford, 1981); E. T. Whittaker and G. N. Watson, A Course of Modern Analysis (Cambridge Univ. Press, Cambridge, 1980).

[5] See, for example, W. Magnus and S. Winkler, Hill's Equation (Wiley, New York, 1966), Ch. 7. 